imperfectly understood in terms of detailed reaction mechanisms. From Stevens's review of the subject there is seen to be much conflicting evidence on the effects of illumination, and further work designed with the object of providing more reliable experimental data is clearly called for.

Taken as a whole, this volume provides a muchneeded co-ordination of recent developments, and will be eagerly studied by all whose work is associated with rubber and polymers, and, it is to be hoped, by others as well.

L. R. G. TreLoar

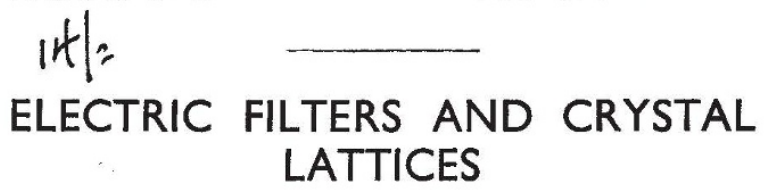

Wave Propagation in Periodic Structures

Electric Filters and Crystal Lattices. By Prof. Léon Brillouin. (International Series in Pure and Applied Physics.) Pp. xii + 247. (New York and London: McGraw-Hill Book Co., Inc., 1946.) 20 s.

TrHIS book deals not with a special branch of 1 physical science but with a general method and its applications 3 Aiferent problems. Its striking feature is the nuther and variety of subjects which are acces iblow the same mathematical treatment : onyong sille problems of pure physics, like scattering of Alays by crystals, thermal vibrations in crystal lattices electronic motion in metals, and on the other side problems of electric engineering, namely, propagation of electro-magnetic waves along periodic circuits and filtering properties of such systems.

In a very attractive introduction the history of the problem is described. The work on periodical structures is as old as modern mechanics itself, since Newton's derivation of the velocity of sound in his "Principia" is based on the consideration of a linear lattice. Many distinguished mathematicians of the eighteenth and nineteenth centuries have written about this subject: the Bernoullis, Taylor, Euler, Lagrange, Cauchy, Baden-Powell and others. Lord Kelvin gave a detailed discussion of the wave propagation in a one-dimensional lattice and discovered all its main properties: the non-linear relation between wave-number and frequency, the existence of a maximum frequency of propagation for systems of equal particles and of several frequency branches for systems of different particles ; in modern terminology, he found the 'filtering' properties of a periodic structure. These results were re-discovered when Einstein's theory of the specific heat of solids demanded a detailed study of crystal lattices.

The succeeding chapters of the book are devoted to a careful presentation of the facts indicated in the introduction. First a mechanical model of particles coupled by elastic springs is used, but soon (Chapter 3) electric structures are considered. Already here, in the linear case, the idea of the reciprocal lattice is introduced. A whole chapter (5) is devoted to the discussion of the velocity and the flow of energy, and the results are expressed in the language of electro-technics by regarding the vibrating system as a transmitter of signals, or as a filter for waves. This way of thinking, familiar to the electrical engineer, is somewhat strange to the physicist. I confess that I have never looked on a lattice as a frequency filter. But this aspect is most interesting, and it is not difficult and most useful to express the results in terms of "passing bands" and "stopping bands", impedance and other such technical expressions.

The propagation of waves in two- and threedimensional lattices is discussed with the help of the reciprocal lattice and of the 'Brillouin zones'. This conception was the main contribution of the author to lattice theory; he has published it in several papers and in a book, "Quantenstatistik" (Springer), and it has been used with great advantage in many investigations. The boundaries of the zones in reciprocal space are the locus of possible discontinuities of the energy distribution. Special cases of this general theorem are Bragg reflexions of X-rays, the energy distribution of electrons in metals and so on. The author applies it also to the theory of specific heat and other thermal properties of solids. This is the only point in the book with which I cannot fully agree. Brillouin derives the distribution law of frequencies of lattice vibrations and shows that each branch contains as many frequencies as the number of cells of the lattice, and he continues (p. 161): "This is a very important and general result of the zone theory". In fact, it follows simply from the existence of the branches and has nothing to do with the zones. This is evident from the fact that it was found many years before the zones were discovered. The generalization of Debye's theory of specific heat based on this theorem (where a separate characteristic temperature is attributed to each branch) dates also from the pre-zone period.

While all the considerations so far are based on approximations (perturbation theory), Chapter 8 is devoted to a study of rigorous solutions in simple cases (Mathieu's and Hill's equation). The last two chapters are the most interesting ones as they give a full account of the author's own work on the propagation of waves along an electric line. Here matrix calculus is applied, and most interesting relations to Pauli's and Dirac's matrices, used in the theory of the spinning electron, are revealed.

The book is delightfully written. The author does not shrink from repeating a formula which has been derived before, so as to save the reader turning over pages. He never says "It is easy to prove" as many writers do (meaning, you have to work hard and spend a considerable time); but he gives the proof clear and simple. It is a work not only for instruction but also for enjoyment. MAx BoRN

\section{MAMMALS OF NEVADA}

\section{Mammals of Nevada}

By E. Raymond Hall. Pp. $x i+710+11$ plates. (Berkeley and Los Ang iv University of Californis Press ; London : Camabluge University Press, 1946.) 42s, net. 7 HIS isfrom by an enthusiast who lives for his throrpuganess of the groundwork on which it is based and the completeness of the treatment that it is unllikely to be superseded. Dr. Hall's knowledge is based on the examination of some eighteen thousand specimens, most of which were collected by expedi. tions led by him between 1930 and 1936, during which he covered the whole State of Nevada.

The first part of the book is devoted to an explana. tion of his methods and to a discussion of general considerations of taxonomic and allied questions: 